\title{
PERFORMANCE ANALYSIS OF MEMS MICROHEATER BY OPTIMIZING COIL DESIGN USING COVENTORWARE
}

\author{
Karan S. Shah ${ }^{1}$, Samiksha R. Gupta ${ }^{2}$, Gauri M. Dalvi ${ }^{3}$, Surendra S. Rathod ${ }^{4}$, Prashant V. Kasambe ${ }^{5}$ \\ Department of Electronics Engineering, Sardar Patel Institute of Technology, Mumbai, India \\ Email : $\left\{{ }^{1}\right.$ karanshh30@gmail.com, ${ }^{2}$ saamiksha.29gupta@gmail.com, ${ }^{3}$ dgauri26@gmail.com, \\ ${ }^{4}$ surendra_rathod@spit.ac.in, ${ }^{5}$ prashant_kasambe@spit.ac.in\}
}

\begin{abstract}
Scaling in VLSI technology is being widely practiced in almost every chip design step. This approach of scaling down things has now found its way in the field of Sensors and actuators which lead to a new path of study viz MEMS (MicroElectro Mechanical System). There are several studies published in the literature which primarily focuses on the design aspect of MEMS sensors; however continuous research is still going on for the perfect sensor structure related for desired application. Microheater is one of the popular MEMS structures that has found its usage in many applications with the most popular being detection of various gases for using higher sensitive detection mechanism at elevated temperature. This paper focuses on design of microheater for various coil structures with CoventorWare. Heating coil being primary element in the microheater, we in this research work have analyzed microheater performance with different coil designs for various materials. The temperature profile of heater coil is studied for different bias voltages. Also for the simulative analysis of heater coil we present a suitable meshing style with appropriate element size for accurate Finite Element Method using CoventorWare.
\end{abstract}

Keywords : MEMS, Gas Detection, Microheater, Coventor Ware

\section{Introduction}

In past, significant research has been carried out for gas sensing with solid state platforms. MEMS based gas sensor platform has been investigated extensively by the worldwide researchers from last decade [1] and was proved to be a potential replacement for ceramic substrate. Semiconducting gas sensing thin film on top of micromachined platform requires an elevated temperature to activate the sensing mechanism. Thus, microheater with fast response and uniform temperature distribution over sensing are of prime concern in case of applications like gas sensing [2]. Researchers have also demonstrated sophisticated low power CMOS compatible Micro Mechanical Systems (MEMS) based sensors [2]. With the increase market trend in the area of Internet of Things (IoT) there is a requirement of wide variety of sensors which can be integrated into CMOS platforms. To integrate sensors in applications like mobile devices we need platform which give real time fast response. Along with the fast response, the other challenges of gas sensing technologies are high energy consumption and fabrication complexity [3].

In India, there are several institutes e.g. IIT Bombay are working on MEMS platforms for various applications one of which is gas sensing. First time in India, Nanosniff Technologies started commercialization of the MEMS based platforms [4]. COMSOL simulation of the Microheater with low power consumption has been reported [5]. To further enhance the research activities in this domain we have initiated the implementation of novel architectures with 3D simulators like ConvetorWare. There are many devices from accelerometer, gyroscope, microphone, optical MEMS, RF MEMS, actuator, energy harvester to resonator are studied with the CoventorWare [6]. We have made an attempt to simulate and analyse microheater for different coil shapes to study the temperature profile for various geometries using CoventorWare and the results are shown in the subsequent sections.

\section{Theoretical Considerations}

MEMS microheaters are micrometer sized hotplates composed of thin suspended membranes that have a resistive heater coil sandwiched between electrically insulating, but thermally conducting layers. Microheaters are intermediate layer of patterned metal micro-structures between insulating layers of silicon dioxide and silicon nitride.

The heart of the microheater is metal coil. All the coil structures are simulated on same microheater substrate for comparative analysis. The size of the 
entire microheater die is $1600 \mu \mathrm{m} \times 1600 \mu \mathrm{m}$. This device size is comaprable to the commercially available microheater from Nanosniff [4] There are two gold contact pads for electrical conductivity. These pads are connected to the heater coil. The heater coil is supplied with an electrical excitation which increases the temperature of the microheater membrane. The structure shown is basically a part of a transducer that requires operation at elevated temperatures for achieving higher sensitivity. These microheater structures must be integrated with a sensitive layer along with electrodes to work as a transducer. Electrodes measure corresponding change in the properties of sensing layer depending upon the principle of transduction.

Various types of designs have been studied in literature with their properties as suitable for a particular operation. S-shaped, double spiral and honeycomb coil structures are selected for this study. They represent different groups that have been formed in the literature as per the characteristics exhibited by them under operating condition [2]. Fig. 1, Fig. 2 and Fig. 3 show the structural design for the Sshaped, double spiral and honeycomb shaped coil microheaters respectively.

These structural designs are prepared in the layout editor of software CoventorWare and serve as a mask for various processes during simulation. The different colors represent mask for different process. The layout editor gives whole picture of all the masks used in the simulation of a particular design by representing them in different colors. These masks can be then used for actual fabrication processes. These masks can also be prepared in autoCAD software and can be imported in a standard gdsII file format. The layout provides us with the $\mathrm{X}$ and $\mathrm{Y}$ dimension planes whereas the $\mathrm{Z}$ dimension is provided by the process editor of Coventoware.

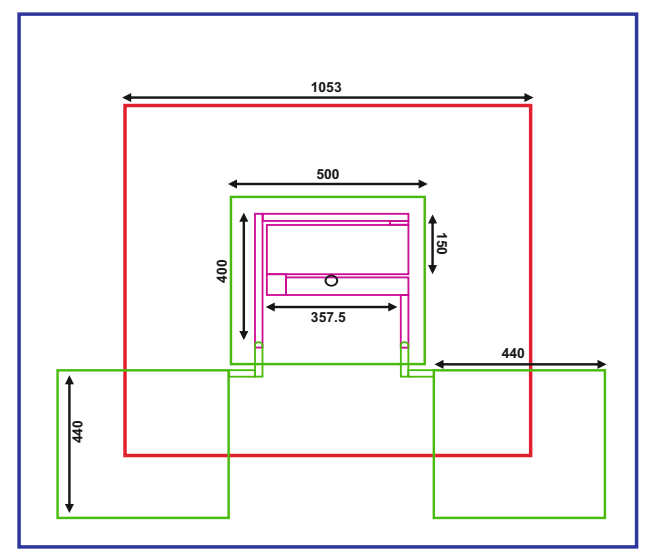

Fig. 1 : Two dimensional layout for the S-shaped coil microheater. (All dimentions are in $\mu \mathrm{m}$, coil thickness $=10 \mu \mathrm{m}$ )

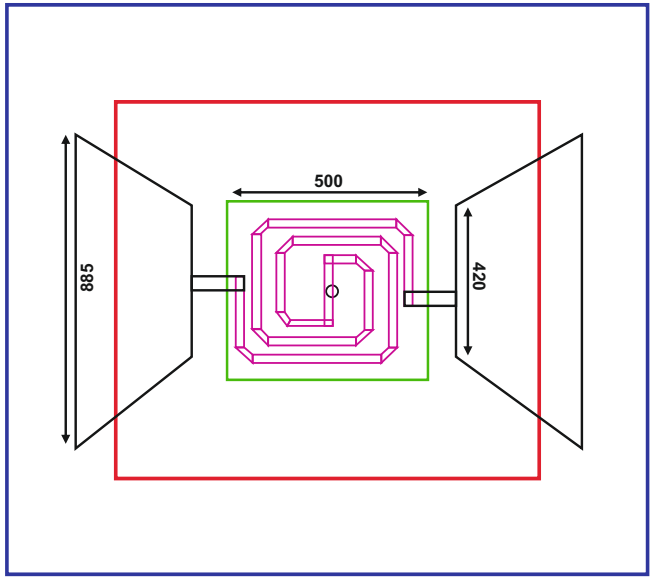

Fig. 2 : Two dimensional layout for the double spiral shaped oil microheater.(All dimentions are in $\mu \mathrm{m}$, coil thickness $=10 \mu \mathrm{m}$ )

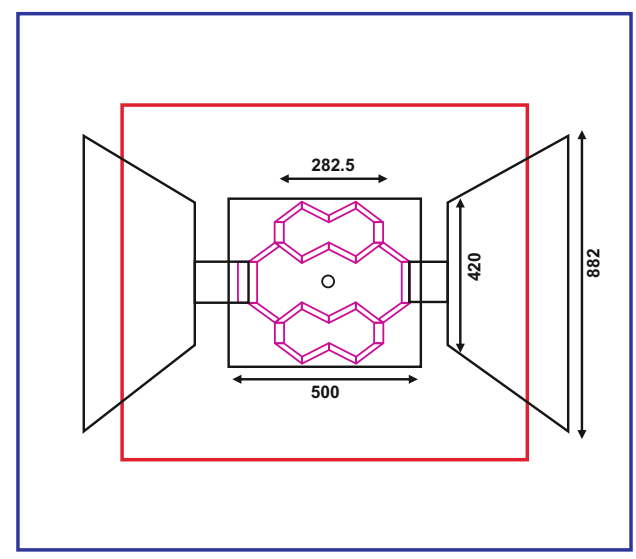

Fig. 3 : Two dimensional layout for the honeycomb shaped coil microheater.(All dimentions are in $\mu \mathrm{m}$, coil thickness $=10 \mu \mathrm{m}$ )

\section{Microheater Modeling and Analysis}

Semiconductor device fabrication process is used to model MEMS micro-heater. It is a multiple step sequence of lithographic and chemical processing steps during which the device is gradually created on a silicon substrate. The CoventorWare allows the simulation of each and every step which resembles the actual process steps [6].

Silicon $<100>$ substrate of $400 \mu \mathrm{m}$ thickness is selected for the microheater configuration. A generic wet etch is then performed on the backside of the substrate to form a tapered sidewall structure with sidewall angle 74.540 . A $0.7 \mu \mathrm{m}$ thick oxide layer is then deposited. Typically the process is modelled by a conformal shell (uniform deposition on exposed surface including trenches). The silicon consumption during oxidation and local oxidation effects such as the bird's beak are not considered in this modeling step. 
After oxidation, a gold layer with $0.2 \mu \mathrm{m}$ thickness is sputtered on the oxidized substrate. This gold layer is then patterned to form 2 different coil structures using mask as given in Fig. 1, Fig. 2 and Fig. 3 with described dimensions.

Following coil design, a sacrificial polyimide layer is deposited on surface for further processes. The thickness of the polyimide layer is chosen as 3um which is sufficient enough to protect gold coil structure from further depositions. Patterning of sacrificial layer is then achieved using gold contact mask with polyimide layer acting as a negative photoresist. Gold layer is then deposited resulting into 2 gold contacts and then patterned for forming 2 gold leads to connect with coil. The sacrificial layer is then stripped off as a final step of the design. Fig. 4, Fig. 5 and Fig. 6 show the final solid model for S-shaped, double spiral and honeycomb shaped coil MEMS microheaters respectively. These solid models show a pictorial representation of MEMS structures and give idea about how the final fabricated device would look like. For analysis of microheater these solid models are then converted into meshed model using mesher settings.

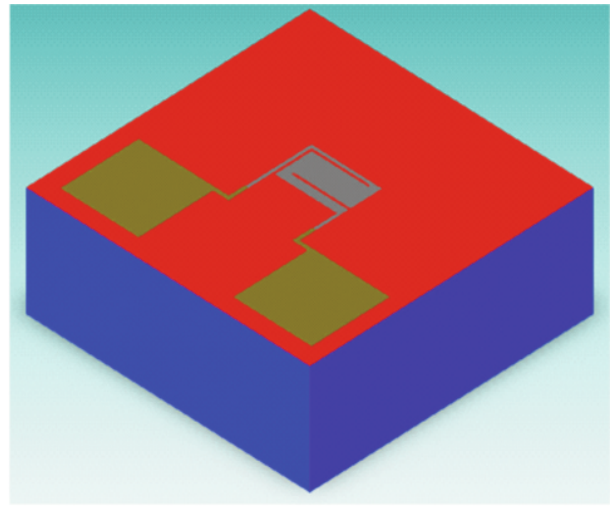

Fig. 4 : Solid model for S-shaped coil microheater

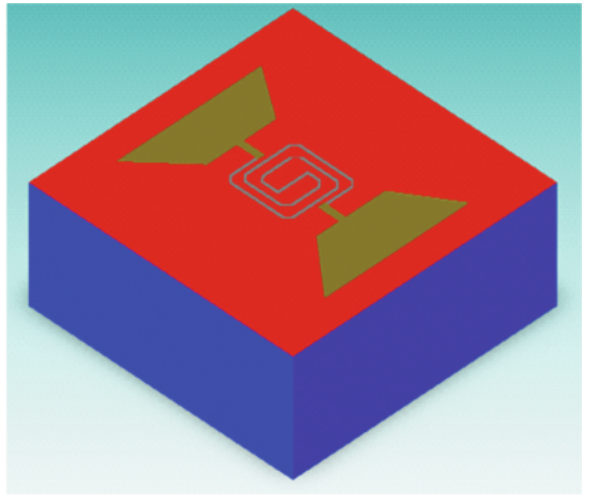

Fig. 5 : Solid model for double spiral shaped coil microheater

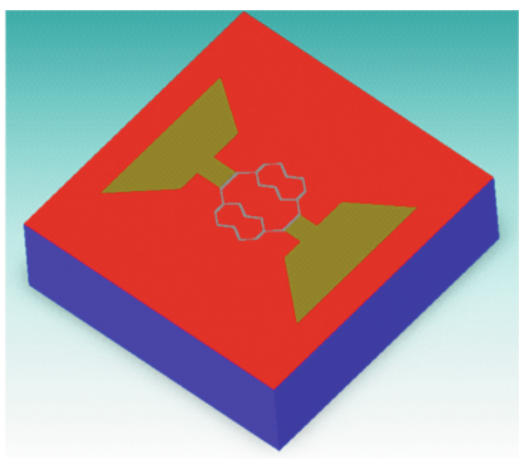

Fig. 6 : Solid model for honeycomb shaped coil microheater

\section{Temperature Profile Analysis}

Electrothermal simulations for the designed microheaters are then carried out using CoventorWare at fixed bias voltage of $5 \mathrm{~V}$. The temperature distribution profiles for the electrothermal simulation are shown in Fig. 7, Fig. 8 and Fig. 9 for S-shaped, double spiral and honeycomb shaped coil microheater respectively. Form the temperature profiles it can be seen that the heating of gold on the oxide layer is limited to coil area and not distributed on the surrounding substrate. This is advantageous in terms of avoiding unwanted heating of electrical contacts and provides fine control of coil temperature as a function of applied bias voltage.

An electrothermal analysis with finite element method (FEM) is used to investigate the thermal properties of electrically driven micro-heaters made up of different materials. The geometric optimization for uniform temperature requirement of micro-heater was performed for wide range of dimensions in CoventorWare. CoventorWare analyzer MemMech solver is used to simulate electrothermal analysis [6].

In this analysis we compute the thermal field and the electrical potential resulting from an imposed voltage and/or current flow through a resistive material using CoventorWare's MemMech module [6]. MemMech's capability is well suited to the design of electrically driven thermal actuators and heat sources. The solver computes the potential through the model based on the voltage applied across the terminals. The coil is set up with an applied voltage, and the potential distribution through the volume is computed. The solver uses the electrical conductivity specified in the model's material properties database in computing the solution. The solver converts the potential values to data points that reflect the Joule heating effects resulting from the current. The solver uses the platinum thermal conductivity specified in the model's material properties database in computing the solution. 


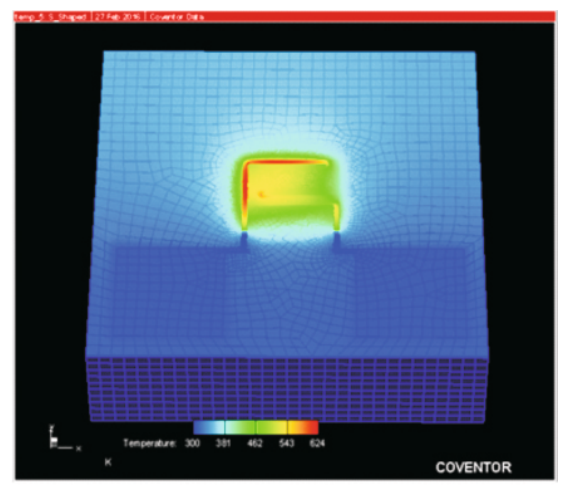

Fig. 7 : Temperature distribution profile for S-shaped coil microheater

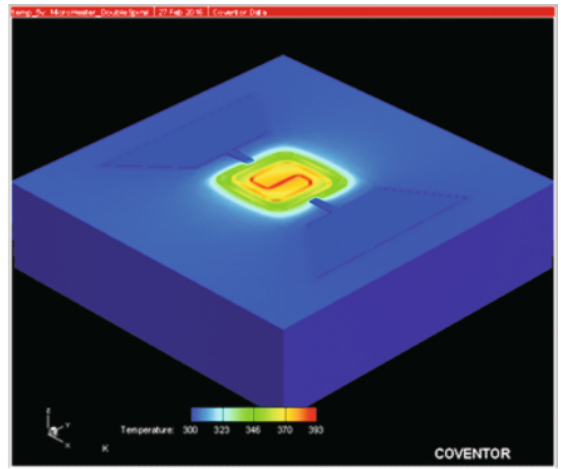

Fig. 8 : Temperature profile distribution for double spiral shaped coil microheater

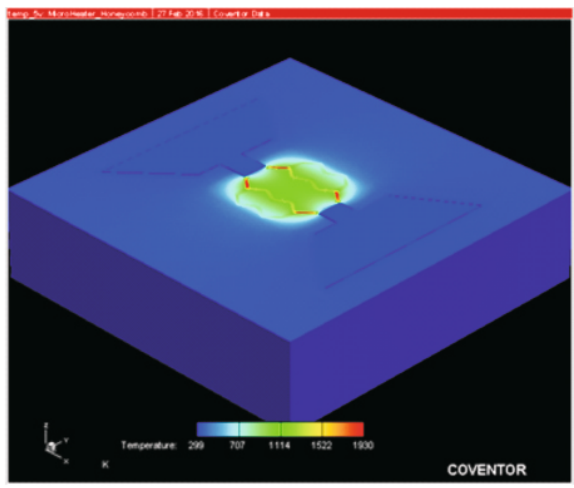

Fig. 9 : Temperature profile distribution for honeycomb shaped coil microheater

\section{Mesh Analysis}

Meshing step in analysis is again one of the important step during Finite element analysis of a model. Meshing is generally used for electrostatic computation and not for mechanical computation. In meshing, solid model is broken into multiple mesh regions and each mesh region is then analysed for electrostatic computation separately. In our research the coil and contact surface is meshed using parabolic extrude while the bottom substrate is meshed using mapped bricks. Since silicon substrate is topologically equivalent to a cube mapped bricks have preferred. Extrude type of meshing is used when the model to be meshed is not having a standard shape. We have used extrude mesh for analyzing the coil and contacts with very small element size for finer meshing in the coil region so as to obtain accurate temperature distribution profile as shown in subsequent section. The mesh size dictates the accuracy of the results obtained in simulation. Fig. 10, Fig 11 and Fig. 12 show meshed model for S-shaped, double spiral and honeycomb shaped coil MEMS microheaters respectively.

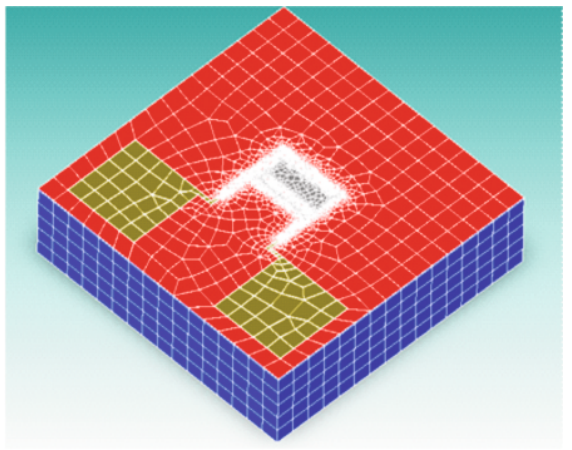

Fig. 10 : Meshed model for S-shaped coil microheater

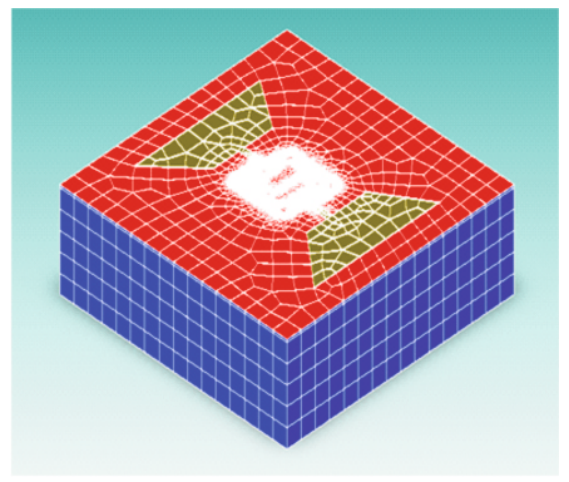

Fig. 11 :Meshed model for double spiral shaped coil microheater

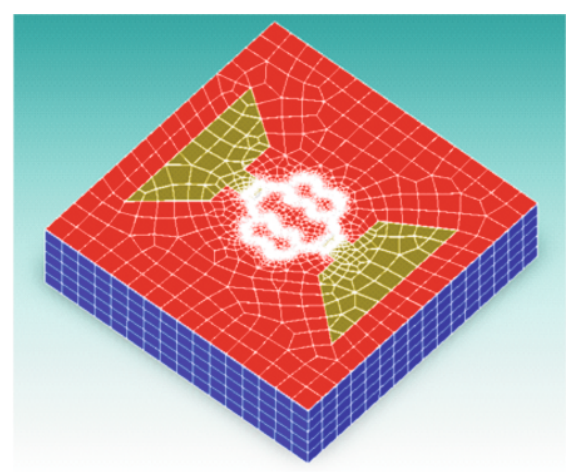

Fig. 12 : Meshed model for honeycomb shaped coil microheater 


\section{Results and Discussion}

Behavior of microheater coils over a series of applied bias voltages is also important for reliable detection by sensing layer. This is because many applications require operation of sensor at various elevated temperatures. MEMS based gas detectors are one of the most widely used application of microheaters that require frequent change of sensor temperatures to detect different gases by using a single sensitive layer. Temperature coefficient of coil material (either positive or negative) is essential for determining the relationship between the applied bias voltages and the required temperature. Thus, for obtaining compatibility of designed microheater structures with gas sensors we have studied maximum temperature attained by coil structures at a given bias. For establishing linear characteristics we have incremented bias voltage from $1 \mathrm{~V}$ to $10 \mathrm{~V}$ in the steps of $1 \mathrm{~V}$. For gold material used in the given coil designs the temperature response for incremental bias voltages is given in Table. 1. Initial coil temperature is $300 \mathrm{~K}$.

Table 1 : Temperature $(\mathrm{K})$ for various coil materials

\begin{tabular}{|c|c|c|c|}
\hline Bias(V). & S-shaped(K) & Double-spiral(K) & Honeycomb(K) \\
\hline 1 & 313 & 304 & 365 \\
\hline 2 & 352 & 315 & 561 \\
\hline 3 & 417 & 333 & 887 \\
\hline 4 & 507 & 359 & 1343 \\
\hline 5 & 624 & 393 & 1930 \\
\hline 6 & 767 & 434 & 2648 \\
\hline 7 & 935 & 482 & 3495 \\
\hline 8 & 1129 & 538 & 4473 \\
\hline 9 & 1350 & 601 & 5582 \\
\hline 10 & 1596 & 672 & 6821 \\
\hline
\end{tabular}

Table. 1 shows the subsequent increase in temperature of all three shapes of heater coil with increase in bias voltage. However, for the same applied bias voltages the increase in temperature of honeycomb shaped coil heater is much greater than the other two designs. This large variation in temerature readings is because of the change in current density. As the cross-sectional area of honeycomb shaped coil microheater is less it gives higher rise in temperature while the double spiral shaped coil microheater having large cross-sectional area gives less increase in coil temperature.
Table 2 : Temperature responses for various mesh sizes

\begin{tabular}{|c|c|c|c|}
\hline $\begin{array}{c}\text { Planar } \\
\text { direction } \\
\text { mesh size for } \\
\text { layer 1 }\end{array}$ & $\begin{array}{c}\text { Planar } \\
\text { direction } \\
\text { mesh size for } \\
\text { heater }\end{array}$ & $\begin{array}{c}\text { Extrude } \\
\text { direction mesh } \\
\text { size for heater }\end{array}$ & $\begin{array}{c}\text { Attained } \\
\text { temperature } \\
\text { (K) }\end{array}$ \\
\hline 500 & 100 & 0.25 & 1694.73 \\
\hline 200 & 20 & 0.20 & 1865.4 \\
\hline 100 & 10 & 0.15 & 1871.13 \\
\hline 50 & 5 & 0.10 & 1930.63 \\
\hline 25 & 2.5 & 0.05 & 1949.62 \\
\hline
\end{tabular}

Table 2 indicates that for very fine mesh in the extrude direction of the Meshed model of the heater results in a more accurate replica of the actual temperature that will be attained by the coil. From the above table, if the mesh size reduces from 500 to 25 , the simulated value of the temperature will be closer to the actual value that would be attained by the heater. If the mesh size decreases by an order of $200 \%$ the actual error in the temperature will be reduced by a factor of $13 \%$. Thus we can have more accurate representation of the actual temperature profile of the device. But a major concern in achieving this will be a large amount of simulation time that would be required to compute very large number of differential and partial equations. Thus a quite a many times it requires careful consideration in obtaining an optimal value between the Mesh size and the amount of time required for simulation.

Many times edge and curvature refinement techniques need to be employed to remove the unwanted part from coming back again and again in the iterations and thus reducing the simulation time thereafter. A best estimate or procedure while choosing an adequate mesh size for a device is to start with a coarse mesh and compare the results with the actual results obtained analytically. If further improvements are required then we can make the mesh size finer and thus achieving a simulative correctresult.

\section{Conclusions}

The study of MEMS microheater is carried out for temperature distribution of heater coil for various coil designs and bias voltages. We have attempted to design and simulate 3 microheaters with CoventorWare which follows the MEMS design process flow. Importance of proper selection of heater coil structure for MEMS microheater based sensor design is emphasized. Further study will be carried out with insulating layer on the heater coil. 


\section{References}

[1] I. Simon, I, N. Barsan, M. Bauer, and U. Weimar, "Micromachined metal oxide gas sensors: Opportunities to improve sensor performance," Sens. Actuators B, Chem., vol. 73, no. 1, pp. 1-26, Feb. 2001.

[2] P. Bhattacharyya "Technological Journey Towards Reliable Microheater Development for MEMS Gas Sensors: A Review," IEEE Trans. on Device and Materials Reliability, Vol. 14, No.2, pp. 589-599, Jun. 2014.

[3] Xiao Liu, Sitian Cheng, Hong Liu, Sha Hu, Daqiang Zhang and Huansheng Ning, "A Survey on Gas Sensing Technology," Sensors, Vol.12, pp. 9635-9665, 2012.
[4] [Online] http://nanosniff.com/

[5] Vineet Bansal, Anil Gurjar, Dinesh Kumar and B. Prasad, "3-D Design, Electro-Thermal Simulation and Geometrical Optimization of spiral Platinum Microheaters for Low Power Gas sensing applications using COMSOL," COMSOL conference, Bangalore, pp. 1-5, 2011.

[6] User Manual, CoventorWare

7] S.S.Mondal , S.Roy , C.K.Sarkar, "Design and Electrothermal analysis of MEMS based Microheater Array for Gas Sensor using INVAR alloy," Inter. Conf. on Communications, Devices and Intelligent Systems (CODIS), pp. 468-471, 2012. 